\title{
Эллипсометрический метод измерения температуры буферных слоев CdTe в технологии молекулярно-лучевой эпитаксии $\mathrm{CdHgTe}$
}

\author{
(C) В.А. Швец ${ }^{1,2}$, И.А. Азаров ${ }^{1,2}$, Д.В. Марин ${ }^{1}$, М.В. Якушев ${ }^{1}$, С.В. Рыхлицкий ${ }^{1}$ \\ ${ }^{1}$ Институт ффизики полупроводников им. А.В. Ржанова Сибирского отделения Российской академии наук, \\ 630090 Новосибирск, Россия \\ ${ }^{2}$ Новосибирский государственный университет, \\ 630090 Новосибирск, Россия \\ E-mail: shvets@isp.nsc.ru
}

(Получена 2 июля 2018 г. Принята к печати 9 июля 2018 г.)

Представлена разработанная эллипсометрическая методика бесконтактного измерения in situ температуры буферного слоя CdTe. Методика основана на температурной зависимости энергетического положения критических точек CdTe и предназначена для определения стартовой температуры поверхности роста перед началом эпитаксии соединения кадмий-ртуть-теллур. Предложен экспрессный метод определения положения критических точек по спектрам эллипсометрического параметра Ч. Проведена серия калибровочных экспериментов, в результате которых получены зависимости положения критических точек от температуры. Оценки и эксперимент показывают, что точность измерения температуры составляет $\pm 3^{\circ} \mathrm{C}$.

DOI: 10.21883/FTP.2019.01.47001.8947

\section{1. Введение}

В настоящее время гетероэпитаксиальная структура на основе КРТ (кадмий-ртуть-теллур) $\mathrm{CdHgTe} / \mathrm{CdTe} / \mathrm{Si}$ является наиболее перспективным материалом для создания инфракрасных фотодетекторов. Она обеспечивает высокий квантовый выход и большую вариативность запрещенной зоны рабочего слоя [1]. Метод молекулярнолучевой эпитаксии (МЛЭ) - один из немногих методов, позволяющих выращивать эпитаксиальные структуры на основе КРТ на подложках из кремния. Это позволяет создавать детекторы в широкой спектральной области от ближнего до дальнего инфракрасного диапазона. Детекторы могут быть как одиночными, так и матричными благодаря равенству коэффициентов термического расширения кремниевой подложки и кремниевой микросхемы считывания сигнала.

Одним из критических параметров технологии МЛЭ КРТ является температура ростовой поверхности. В используемых для роста установках МЛЭ отсутствует тепловой контакт между держателем образца, самим образцом и нагревателем. Поэтому нагрев подложки осуществляется тепловым излучением. Его мощность регулируется напряжением на нагревательном элементе, температура которого контролируется термопарой. Такая схема нагрева позволяет оперативно проводить загрузку и выгрузку образца без разгерметизации рабочего объема, но делает практически невозможным измерение его температуры термопарным методом. Во-первых, технически крайне сложно обеспечить надежный тепловой контакт термопары с образцом при частой загрузке подложек. Во-вторых, такой контакт будет создавать дополнительный канал теплообмена и, как следствие, приводить к образованию градиента температуры по площади образца в области контакта [2]. Измерение температуры держателя образца не решает поставлен- ную задачу ввиду того, что температуры держателя и самого образца заметно различаются из-за слабого теплового контакта и различий в их излучательных способностях $[3,4]$.

Альтернативный путь решения проблемы - использование для измерения температуры бесконтактных методов, таких как: пирометрия [3,5], дифракция быстрых электронов [6], спектроскопия поглощения света [7], эллипсометрия [8-10]. Каждый из этих методов имеет свои особенности и преимущества. Применительно к технологии МЛЭ КРТ метод пирометрии из-за низкой температуры роста оказывается сложным как в технической реализации, так и в методическом плане. Дифракция быстрых электронов применяется для контроля температуры на ограниченном числе объектов. Наиболее предпочтительными являются оптические методы, в частности эллипсометрия. С помощью эллипсометра можно измерять диэлектрические функции и определять зависящие от температуры положения критических точек. С целью решения задачи термометрии с одновременным определением состава в работе [9] были измерены спектры КРТ и создана параметрическая модель диэлектрических функций, учитывающая зависимости от состава и температуры. Аналогичные исследования были выполнены также для CdZnTe [10]. Следует, однако, отметить, что одновременный мониторинг температуры и состава соединения представляется сложной задачей как в экспериментальном, так и в методическом плане.

Более простой, но не менее важной для технологии МЛЭ КРТ задачей является прецизионное измерение температуры буферного слоя CdTe для задания стартовых условий перед началом эпитаксии КРТ. Ранее нами были сделаны оценки возможности метода одноволновой эллипсометрии для измерения температуры CdTe на длине волны излучения $\mathrm{He}-\mathrm{Ne}$-лазера [11]. Оценки показали, что точность определения температуры при вы- 
полнении ряда технических требований может составить в лучшем случае $10-20^{\circ} \mathrm{C}$, что явно недостаточно для воспроизводимой технологии. В данной работе предлагается спектральная эллипсометрическая методика измерения температуры CdTe, основанная на температурной зависимости положений критических точек плотности состояний (особенностей Ван-Хова). Для этого нами были проведены измерения ex situ образцов и определены калибровочные зависимости длины волны критических точек от температуры. Наибольший интерес в этом отношении представляет дублет $E_{1} /\left(E_{1}+\Delta_{1}\right)$ (длины волн $\lambda=370 / 314 \mathrm{Hм})$ и край поглощения $E_{0}=1.5$ эВ $(\lambda \approx 830$ нм $)$, которые попадают в спектральный диапазон оптической эллипсометрии $(\lambda=250-1000 \mathrm{Hм})$.

\section{2. Подготовка образцов и измерения}

Слои СdTе толщиной $d \approx 6$ мкм на подложке $\mathrm{Si}$ были выращены в многокамерной сверхвысоковакуумной установке молекулярно-лучевой эпитаксии „Обь“ [12], детали технологического процесса, приведены в работе [13]. В качестве подложек использовались кремниевые пластины диаметром 76.2 мм, ориентированные в плоскости (310).

Для измерения эллипсометрических спектров использовался ex situ эллипсометр статического типа, описанный в работе [14]. Диапазон длин волн эллипсометра 250-1100нм, шаг сканирования по спектру 2 нм при спектральном разрешении 3 нм. Калибровка монохроматора проводилась по линиям излучения ртутной и ксеноновой ламп, а также по линии излучения $\mathrm{He}-\mathrm{Ne}-$ лазера. Воспроизводимость измерения эллипсометрических параметров составляла $\delta \Psi=0.02^{\circ}$ и $\delta \Delta=0.05^{\circ}$, угол падения света на образец равнялся $65^{\circ}$. Время измерения одного спектра составляло $20 \mathrm{c}$.

Прогрев образцов осуществлялся с помощью малогабаритной печки, которая представляла собой медную шайбу, нагреваемую спиралью с протекающим по ней стабилизированным током. Исследуемый образец прижимался к поверхности шайбы пружиной. Для создания теплового контакта поверхность образца смачивалась индий-галлиевой эвтектикой. Температура образца измерялась с помощью термопары хромель-алюмель, спай которой располагался в углублении медной шайбы вблизи образца и также смачивался эвтектикой. Для достижения теплового равновесия нагреватель в течение нескольких минут перед измерением выдерживался при фиксированной температуре $T$. Точность измерения температуры оценивается нами как $\pm 3^{\circ} \mathrm{C}$.

Все измерения проводились на открытом воздухе в диапазоне температур от комнатной до $222^{\circ} \mathrm{C}$. Взаимодействие с воздухом может привести к образованию окисных слоев или другим нежелательным изменениям на поверхности, которые будут влиять на измеряемые спектры. Поэтому предварительно проводились исследо-

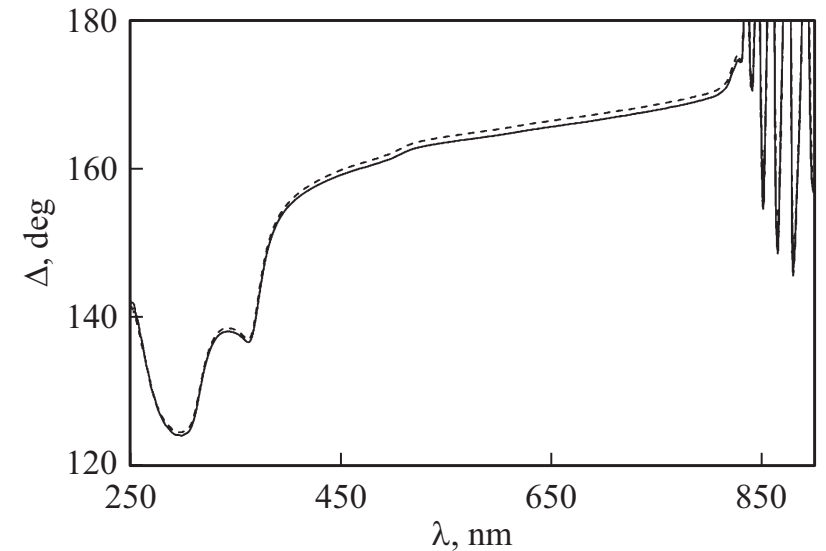

Рис. 1. Спектры $\Delta(\lambda)$ для образца СdTе, измеренные при комнатной температуре: сплошная линия - до отжига, штриховая - после нагрева до $220^{\circ} \mathrm{C}$.

вания стабильности поверхности СdTе при прогревах на открытом воздухе.

Было установлено, что после первого прогрева образцов до $220^{\circ} \mathrm{C}$ и охлаждения до комнатной температуры спектры эллипсометрических параметров изменяются (рис. 1). Для спектра $\Psi(\lambda)$ эти изменения незначительные и составляют $\sim 0.1^{\circ}$, а в спектре $\Delta(\lambda)$ наблюдается смещение в сторону более высоких значений $\Delta$, которое в диапазоне 400-800 нм достигает величины $1^{\circ}$. При последующих циклах нагрева и остывания зависимости $\Psi(\lambda)$ и $\Delta(\lambda)$, измеренные при комнатной температуре, остаются неизменными. Такое поведение эллипсометрических спектров можно интерпретировать как удаление с поверхности образца при первом прогреве адсорбированных слоев, предположительно воды. Заметим, что аналогичные эффекты наблюдались также при первых нагревах слоев КРТ [15] и наноструктур на основе кремния [16]. Таким образом, можно заключить, что после первого прогрева образцов CdTe на воздухе дальнейшие его нагревы в указанном температурном интервале не приводят к окислению поверхности или другим нежелательным процессам, влияющим на эллипсометрические спектры.

\section{3. Экспериментальные результаты и обсуждение}

На рис. 2 показаны зависимости $\Psi(\lambda)$ вблизи $E_{1}$ и $\left(E_{1}+\Delta_{1}\right)$, измеренные в диапазоне температур от 25 до $222^{\circ} \mathrm{C}$. Наблюдается монотонная трансформация спектров и смещение максимумов с ростом температуры. Положения максимумов $\Psi\left(\lambda_{\max }\right)$, соответствующих $E_{1}$ и $\left(E_{1}+\Delta_{1}\right)$ по шкале длин волн, в зависимости от температуры показаны на рис. 3 для нескольких циклов нагрева и остывания. Данные рисунка хорошо описываются линейными зависимостями с наклонами $k_{1}=0.072 \mathrm{HM} /{ }^{\circ} \mathrm{C}$ и $k_{2}=0.041 \mathrm{Hм} /{ }^{\circ} \mathrm{C}$ для $E_{1}$ и $\left(E_{1}+\Delta_{1}\right)$ 


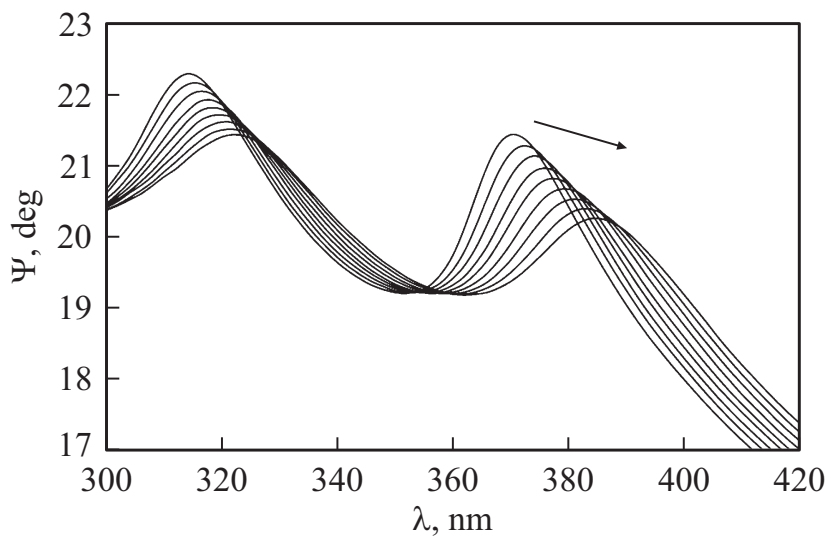

Рис. 2. Фрагмент спектров параметра $\Psi$, измеренных при пошаговом увеличении температуры от 25 до $222^{\circ} \mathrm{C}$. Стрелкой показано направление смещения спектров при увеличении температуры. Все кривые эквидистантны по температуре.

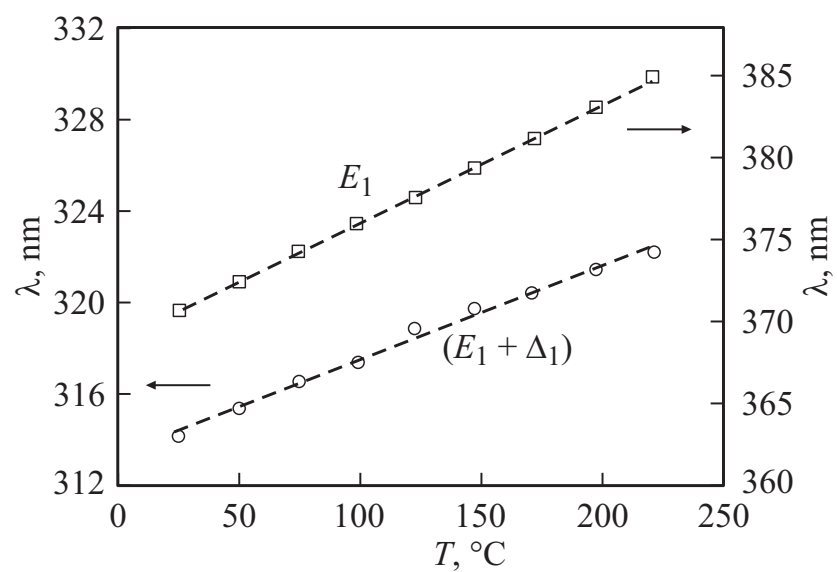

Pис. 3. Зависимость положения максимумов в спектрах $\Psi(\lambda)$ от температуры вблизи критических точек $E_{1}$ и $\left(E_{1}+\Delta_{1}\right)$.

соответственно, которые определяют чувствительность $\lambda_{\max }$ к изменению температуры. Найденные коэффициенты чувствительности близки к значениям 0.081 и $0.055 \mathrm{Hм} /{ }^{\circ} \mathrm{C}$, полученным для $E_{1}$ и $\left(E_{1}+\Delta_{1}\right)$ в работе [17]. Следует отметить, что в цитируемой работе приведена зависимость положений критических точек, которые совпадают с максимумами диэлектрической функции $\varepsilon_{2}$, в то время как на рис. 3 изображена зависимость для максимумов $\Psi$. Точность, с которой удается найти положение максимума, определяется погрешностью эллипсометрических измерений. Положение максимума рассчитывалось путем аппроксимации зависимости $\Psi(\lambda)$ кубической параболой, параметры которой находились методом наименьших квадратов. Если использовать достаточно большое число экспериментальных точек, 7-8, то длина волны максимума определяется с точностью $\delta \lambda= \pm 0.2$ нм. Таким образом, измеряя спектр $\Psi(\lambda)$ вблизи $E_{1}$, мы можем рассчитывать на точность определения температуры $\delta T=\delta \lambda / k_{1} \approx \pm 3^{\circ} \mathrm{C}$.
Для максимума $\left(E_{1}+\Delta_{1}\right)$ чувствительность к температуре $k_{2}$ ниже, к тому же разброс экспериментальных данных заметно больше, чем для аналогичного максимума $E_{1}$. При измерениях in situ разброс данных может оказаться еще больше из-за запыления окон, которое приводит к уменьшению интенсивности света, в первую очередь в коротковолновой области. Поэтому возможность использования для решения поставленной задачи максимума, соответствующего $\left(E_{1}+\Delta_{1}\right)$, представляется менее привлекательной.

Наряду со спектральной зависимостью максимумов $\Psi$ можно рассматривать положения максимумов в спектре мнимой части диэлектрической функции $\varepsilon_{2}(\lambda)$, которые также ассоциируются с критическими точками. Однако следует учитывать, что при вычислении $\varepsilon_{2}$ используются оба измеренные эллипсометрические параметра: $\Psi$ и $\Delta$. При этом фазовый параметр $\Delta$ в большей степени чувствителен к несовершенству поверхности (например, к микрошероховатости, выходу дефектов кристаллической структуры и т.п.). Кроме того, точность измерения параметра $\Delta$ ниже, чем параметра $\Psi$. Поэтому экспериментальная точность определения максимума $\varepsilon_{2}$ оказывается ниже, чем для $\Psi$.

Кроме области критических точек $E_{1}$ и $\left(E_{1}+\Delta_{1}\right)$, большой интерес представляет область вблизи края поглощения, где начинает проявляться интерференция эллипсометрических параметров. На рис. 4 показаны фрагменты двух спектров $\Psi(\lambda)$, измеренных при температурах 25 и $222^{\circ} \mathrm{C}$. Начало интерференционных осцилляций по спектру соответствует краю поглощения $\lambda_{0}$, который отмечен для каждой кривой вертикальной стрелкой. Наблюдается значительное смещение края с ростом температуры, которое превышает $40 \mathrm{Hм}$ на интервале $200^{\circ} \mathrm{C}$. Столь высокая температурная чувствительность делает рассматриваемую область спектра привлекательной для реализации температурного контроля. Для этого необходимо решить чисто мето-

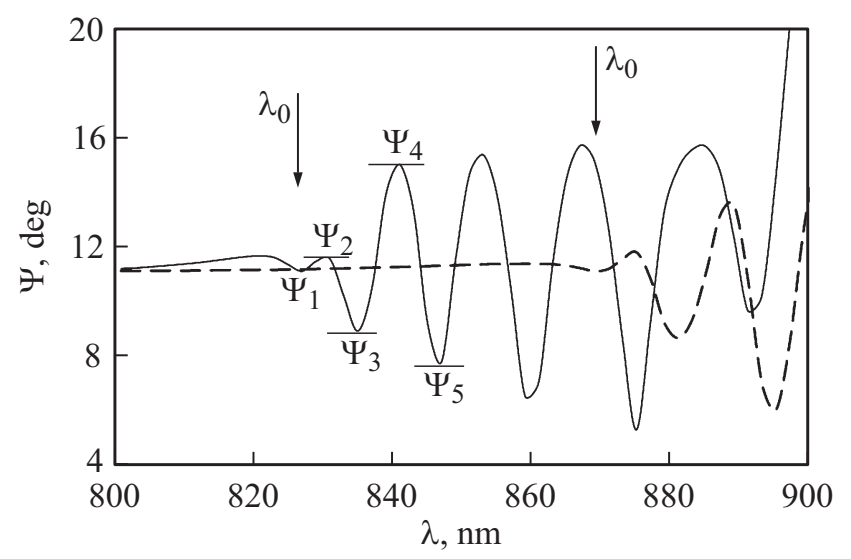

Pис. 4. Спектры $\Psi(\lambda)$ вблизи края поглощения CdTe, измеренные при температуре 25 (сплошная линия) и $222^{\circ} \mathrm{C}$ (штриховая). Вертикальными стрелками показаны положения края поглощения. Горизонтальными линиями отмечены положения интерференционных экстремумов. 
дический вопрос и разработать критерий определения длины волны $\lambda_{0}$ по измеренным спектрам. Наиболее корректный подход - это численное решение обратной эллипсометрической задачи, т.е. определение толщины слоя CdTe и восстановление спектральных зависимостей показателей преломления и поглощения. Однако такой подход оказывается достаточно громоздким, так как решение обратной задачи обычно предполагает интерактивное участие оператора, поэтому его реализация в режиме реального времени в процессе роста затруднена.

Нами предложен эмпирический метод нахождения $\lambda_{0}$. Амплитуда интерференционных осцилляций параметра $\Psi$ в некотором достаточно узком диапазоне длин волн, соответствующем области Урбаха (т.е. при $\lambda>\lambda_{0}$ ), монотонно возрастает с увеличением длины волны. На рисунке горизонтальными линиями отмечены ближайшие к $\lambda_{0}$ интерференционные экстремумы в спектре $\Psi(\lambda)$. Разница между соседними экстремумами по модулю $\delta \Psi=\left|\Psi_{j}-\Psi_{j+1}\right|$ характеризует среднее значение удвоенной амплитуды осцилляций на интервале между $j$-м и $(j+1)$-м экстремумами. На рис. 5 изображены полученные таким образом зависимости $\delta \Psi(\lambda)$ при различных температурах: экспериментальные данные и их аппроксимация линейными функциями.

На выбранном интервале длин волн эти зависимости хорошо ложатся на прямые, пересечение которых с горизонтальной осью можно в каждом случае принять за $\lambda_{0}$. Количество точек, которые попадают на линейный участок, зависит от частоты осцилляций и в конечном итоге от толщины слоя CdTe. В отработанной технологии выращивания структур при толщине буферного слоя 6 мкм оптимальное количество точек для построения линии аппроксимации оказывается равным 4. При увеличении до 5 или 6 наблюдается насыщение амплитуды и отклонение от линейной зависимости. Предложенный

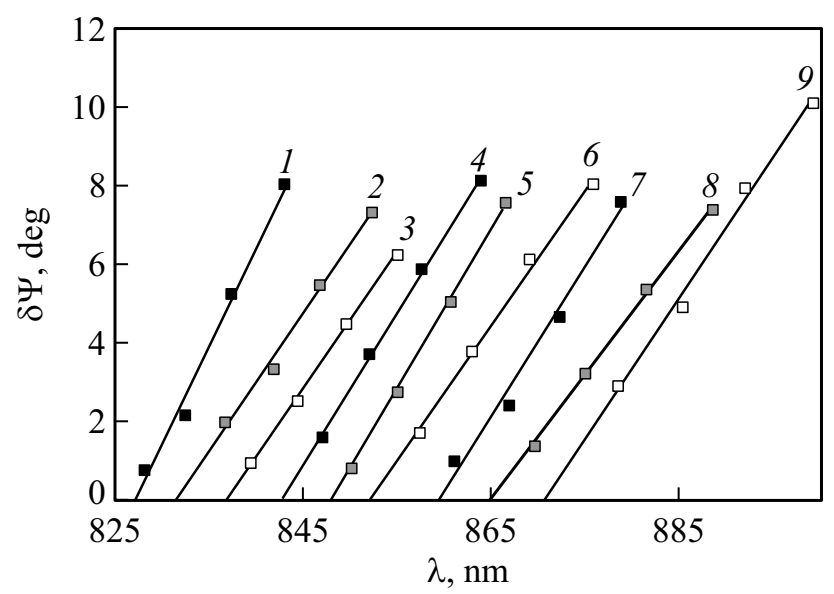

Рис. 5. Зависимости амплитуды осцилляции $\delta \Psi(\lambda)$, полученные из спектров интерференции $\Psi(\lambda)$ при различных температурах. Точки - экспериментальные данные, прямые линии аппроксимация. Температура, ${ }^{\circ} \mathrm{C}: 1-25,2-50,3-74$, $4-99,5-123,6-148,7-172,8-198,9-222$.

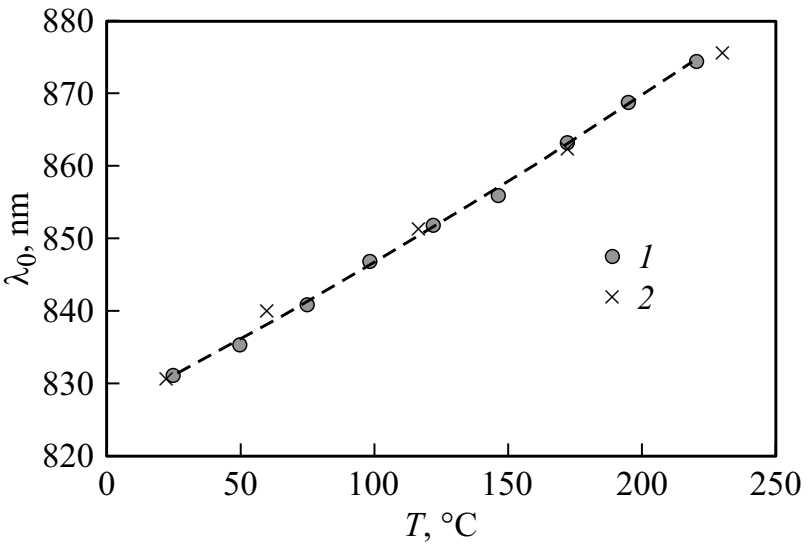

Рис. 6. Зависимость края поглощения $\lambda_{0}$ от температуры CdTe. 1 - эксперимент, 2 - результаты, полученные пересчетом данных, приведенных в работе [17].

алгоритм вычисления $\lambda_{0}$ достаточно прост и может быть легко реализован в реальном времени.

Зависимость найденных таким образом значений $\lambda_{0}$ от температуры нагрева показана на рис. 6. Величина $\lambda_{0}$ практически линейна по температуре. Для сравнения на рисунке приведены также аналогичные результаты, пересчитанные из данных работы [17], которые практически совпадают с нашими измерениями. B paботах S. Adachi $[18,19]$ в качестве энергии критической точки, соответствующей краю поглощения, указывается $E_{0}=1.5$ эВ при $T=300 \mathrm{~K}$, что соответствует $\lambda_{0}=827$ нм и несколько отличается от данных работы [17] и наших данных. Такое отличие может быть связано с разными критериями определения $E_{0}$ и $\lambda_{0}$. Существенно, однако, что наклон для обеих групп данных, представленных на рис. 6, который определяет чувствительность $\lambda_{0}$ к температуре, оказывается практически одинаковым.

Оценим точность метода. Она определяется приведенной выше температурной чувствительностью $\lambda_{0}(T)$ и экспериментальной точностью нахождения величины $\lambda_{0}$. В таблице приведена статистика измерений величины $\lambda_{0}$, выполненных при комнатной температуре на серии образцов $\mathrm{Si} / \mathrm{CdTe}$ и $\mathrm{GaAs} / \mathrm{CdTe}$ с приблизительно одинаковой толщиной слоя СdTe $d \approx 6$ мкм. Для $\sim 65 \%$ измерений разброс значений $\lambda_{0}$ не превышает \pm 0.5 нм. Для оставшихся 35\% величина $\lambda_{0}$ имеет более значительные отклонения и смещена в сторону меньших длин волн, т.е. статистическое распределение не является симметричным. Интересно отметить, что для той части выборки, которая имеет большое отклонение $\lambda_{0}$ (в таблице эти данные выделены жирным шрифтом), наблюдается меньший наклон прямых $\delta \Psi(\lambda)$, показанных на рис. 5. Тангенс угла наклона приведен в таблице (параметр $\alpha$ ). Величина $\alpha$ показывает скорость нарастания интерференционных осцилляций при возрастании $\lambda$, т.е. степень размытия края поглощения, что в свою очередь может быть связано с дефектностью пленок CdTe. 


\begin{tabular}{c|c|c|c}
\hline Номер образца & Подложка & $\lambda_{0}$, нм & $\alpha$, град/нм \\
\hline 1 & $\mathrm{Si}$ & 831.6 & 0.349 \\
2 & $\mathrm{Si}$ & 830.8 & 0.331 \\
3 & $\mathrm{Si}$ & $\mathbf{8 3 0 . 0}$ & $\mathbf{0 . 2 9 5}$ \\
4 & $\mathrm{Si}$ & $\mathbf{8 2 9 . 2}$ & $\mathbf{0 . 2 9 7}$ \\
5 & $\mathrm{Si}$ & 830.3 & 0.306 \\
6 & $\mathrm{Si}$ & 831.2 & 0.374 \\
7 & $\mathrm{Si}$ & 830.9 & 0.368 \\
8 & $\mathrm{Si}$ & $\mathbf{8 2 9 . 3}$ & $\mathbf{0 . 3 1 6}$ \\
9 & $\mathrm{Si}$ & 830.6 & 0.342 \\
10 & $\mathrm{GaAs}$ & 831.1 & 0.553 \\
11 & $\mathrm{GaAs}$ & 831.2 & 0.533 \\
12 & $\mathrm{GaAs}$ & $\mathbf{8 2 9 . 1}$ & $\mathbf{0 . 3 8 0}$ \\
13 & $\mathrm{GaAs}$ & 830.9 & 0.493 \\
14 & $\mathrm{GaAs}$ & $\mathbf{8 2 8 . 6}$ & $\mathbf{0 . 3 7 1}$ \\
15 & $\mathrm{GaAs}$ & 831.1 & 0.449 \\
16 & $\mathrm{GaAs}$ & 831.2 & 0.499 \\
17 & $\mathrm{GaAs}$ & 830.8 & 0.463
\end{tabular}

Если для оценки точности в качестве экспериментальной ошибки взять величину $\delta \lambda_{0}=0.5$ нм, то погрешность измерений температуры составит $\pm 3^{\circ}$, что совпадает с приведенной выше оценкой точности измерения температуры по положению пика $E_{1}$. На практике, повидимому, целесообразно объединить оба эти подхода и определять температуру комплексным образом, учитывая данные обоих спектральных диапазонов.

Помимо случайного статистического разброса, который можно уменьшить путем многократных измерений спектров, определяемое значение температуры зависит также от ряда факторов, приводящих к систематическим ошибкам, таких как: качество кристаллической структуры CdTe, образование в процессе роста поверхностной шероховатости, невоспроизводимость угла падения света и другие. Проведенные нами измерения $\lambda_{0}(T)$ на образцах с различной поверхностной плотностью прорастающих дефектов не выявили каких-либо различий для калибровочных зависимостей, представленных на рис. 3 и 6. Поэтому можно утверждать, что дефектность слоев $\mathrm{CdTe}$, появляющаяся в пределах естественного технологического разброса, не дает сколь-нибудь заметного эффекта.

Второй источник систематической ошибки - возможная микрошероховатость поверхности растущих слоев, которая сказывается на измеряемых эллипсометрических спектрах. В процессе роста степень шероховатости контролируется с помощью встроенного одноволнового эллипсометра. Образование шероховатости приводит к уменьшению фазового эллипсометрического параметpa $\Delta[20]$. В оптическом смысле шероховатость эквивалентна поверхностному слою, поэтому ее влияние легко можно промоделировать численно. Проведенные расчеты показывают, что появление шероховатости в тех масштабах, которые наблюдаются в ростовых экспериментах, приводит к сдвижке максимумов параметра $\Psi$ для $E_{1}$ и $\left(E_{1}+\Delta_{1}\right)$ на величину $\sim(0.1-0.2)$ нм и не превышает экспериментальной точности определения положения этих максимумов.

Невоспроизводимость угла падения $\delta \varphi= \pm 0.05^{\circ}$ служила одной из причин, которая ограничивала точность измерения температуры в методе одноволновой эллипсометрии. При изменении угла падения эллипсометрические спектры также изменяются, но положения максимумов $\Psi$ по шкале длин волн остаются практически неизменными. То же самое относится и к значению $\lambda_{0}$, которое определяется по началу интерференционных осцилляций. Здесь следует отметить, что рассматриваемая методика устойчива по отношению к систематическим приборным погрешностям, за исключением погрешности калибровки монохроматора.

\section{4. Заключение}

В работе рассмотрена одна из актуальных проблем вакуумной технологии МЛЭ КРТ - проблема контроля температуры поверхности роста. В рамках решения этой проблемы разработана и представлена комплексная эллипсометрическая методика измерения температуры буферного слоя CdTe перед началом эпитаксиального роста КРТ. Методика основана на измерении спектров эллипсометрических параметров и определении энергетических положений температурно-зависимых особенностей спектра, соответствующих критическим точкам материала. Температурные измерения ex situ, проведенные для $\mathrm{CdTe}$ в диапазоне $25-222^{\circ} \mathrm{C}$, показывают, что точность определения температуры составляет $\pm 3^{\circ} \mathrm{C}$. Предложенная методика после дополнительных калибровочных экспериментов может быть распространена и на другие материалы.

\section{Список литературы}

[1] E. Finkman, S. E. Schacham. J. Appl. Phys., 56, 2896 (1984).

[2] О.А. Геращенко. Температурные измерения. Справочник (Киев, Наук. думка, 1989).

[3] И.А. Азаров, В.А. Швец, С.А. Дулин, Н.Н. Михайлов, С.А. Дворецкий, Д.Г. Икусов, И.Н. Ужаков, С.В. Рыхлицкий. Автометрия, 53 (6), 111 (2017).

[4] M. Kawano, T. Sasaki, N. Oda. J. Electron. Mater., 24 (5), 431 (1995).

[5] Z.M. Zhang, B.K. Tsai, G. Machin. Radiometric temperature measurements. II Applications. Experimental methods in the physical sciences (Amsterdam-Boston-Elsevier, Academic Press, 2010).

[6] A.J. SpringThorpe, S.J. Ingrey, B. Emmerstorfer, P. Mandeville. Appl. Phys. Lett., 50, 77 (1987).

[7] D. Wahl, M. Mertens. Time-Resolved In-Situ Temperature Measurements Using Band-Edge Absorption Spectroscopy During MBE Growth. Annual Report 2010 (Institute of Optoelectronics, Ulm University).

[8] T. Tomita, T. Kinosada, T. Yamashita, M. Shiota, T. Sakurai. Jpn. J. Appl. Phys., 25, L925 (1986).

[9] B. Johs, C.M. Herzinger, J.H. Dinan, A. Cornfeld, J.D. Benson. Thin Sol. Films, 313-314, 137 (1998). 
[10] M. Daraselia, G. Brill, J.W. Garland, V. Nathan, S. Sivananthan. J. Electron. Mater., 29, 742 (2000).

[11] И.А. Азаров, Д.В. Марин, В.А. Швец, М.В. Якушев. Тез. докл. Росс. конф. по актуальным проблемам полупроводниковой фотоэлектроники (Новосибирск, 2017) c. 119.

[12] Ю.Г. Сидоров, С.А. Дворецкий, Н.Н. Михайлов, М.В. Якушев, В.С. Варавин, А.П. Анциферов. Оптич. журн., 67, 39 (2000).

[13] М.В. Якушев, Д.В. Брунев, В.С. Варавин, В.В. Васильев, С.А. Дворецкий, И.В. Марчишин, А.В. Предеин, И.В. Сабинина, Ю.Г. Сидоров, А.В. Сорочкин. ФТП, 45 (3), 396 (2011).

[14] Е.В. Спесивцев, С.В. Рыхлицкий, В.А. Швец. Автометрия, $47(5), 5$ (2011).

[15] Г.Ю. Сидоров, В.А. Швец, Ю.Г. Сидоров, В.С. Варавин. Автометрия, 53 (6), 97 (2017).

[16] E.V. Spesivtsev, S.V. Rykhlitsky, V.A. Shvets, S.I. Chikichev, A.S. Mardezhov, N.I. Nazarov, V.A. Volodin. Thin Sol. Films, 455-456, 700 (2004).

[17] C.C. Kim, M. Daraselia, J.W. Garland, S. Sivananthan. Phys. Rev. B, 56 (8), 4786 (1997).

[18] S. Adachi, T. Kimura, N. Suzuki. J. Appl. Phys., 74 (5), 3435 (1993).

[19] S. Adachi. Optical constants of crystalline and amorphous semiconductors: Numerical data and graphical information (Kluwer Academic Publishers, Boston-Dresden-London, 1999).

[20] K.K. Svitashev, V.A. Shvets, A.S. Mardezhov, S.A. Dvoretsky, Yu.G. Sidorov, N.N. Mikhailov, E.V. Spesivtsev, S.V. Rychlitsky. Mater. Sci. Engin., B44, 162 (1996).

Редактор Л.В. Шаронова

\title{
Ellipsometric method for measuring of the CdTe buffer layer temperature in molecular beam epitaxy of CdHgTe
}

\author{
V.A. Shvets ${ }^{1,2}$, I.A. Azarov ${ }^{1,2}$, D.V. Marin ${ }^{1}$, \\ M.V. Yakushev' ${ }^{1}$, S.V. Rykhlitsky ${ }^{1}$ \\ ${ }^{1}$ Rzhanov Institute of Semiconductor Physics, \\ Siberian Branch of Russian Academy of Sciences, \\ 630090 Novosibirsk, Russia \\ 2 Novosibirsk State University, \\ 630090 Novosibirsk, Russia
}

Abstract The paper presents the developed ellipsometric technique of non-contact in situ measurement of the CdTe buffer layer temperature. The technique is based on the temperature dependence of the energy position of CdTe critical points and is designed to determine the starting temperature of the growth surface before epitaxy of the cadmium-mercury-telluride compound. An express method for determining the position of critical points in the spectra of the ellipsometric $\Psi$ parameter is proposed. A series of experiments were carried out, which resulted in determination of the position of critical points on temperature. Estimates and experiment show that the temperature measurement accuracy is $\pm 3^{\circ} \mathrm{C}$. 